\title{
Punción aspiración guiada por ultrasonido vs artroscopia en la resolución de tendinopatía cálcica del manguito de los rotadores de hombro
}

\author{
Ultrasound-guided aspiration puncture vs arthroscopy of the \\ shoulder rotator cuff calcic tendinopathy resolution
}

\author{
Juan Sarasquete Reiris, ${ }^{*}$ Luis Gerardo Domínguez Gasca, ${ }^{\ddagger}$ Carlos Lobo Oropeza ${ }^{\S}$ \\ Citar como: Sarasquete RJ, Domínguez GLG, Lobo OC. Punción aspiración guiada por ultrasonido vs artroscopia en la resolución de tendinopatía \\ cálcica del manguito de los rotadores de hombro. Acta Med Grupo Angeles. 2021; 19 (2): 180-185. https://dx.doi.org/10.35366/100439
}

\section{Resumen}

Introducción: La tendinopatía calcificante de hombro es una de las principales causas de omalgia de origen no traumático en las personas entre 50-60 años de edad, ocasionando discapacidad. Objetivo: Comparar la efectividad terapéutica de la punción y aspiración guiada por ecografía (grupo A) con la reparación vía artroscópica (grupo B). Material y métodos: 32 pacientes atendidos entre febrero de 2017 y febrero de 2019; 17 pacientes conformaron el grupo A y 15 el grupo B, valorando resultados con mejoría clínica y radiológica y escalas de funcionalidad del hombro (CONSTANT y DASH) obtenidos previo a la intervención, a los seis meses y al año. Resultados: No se encontró diferencia estadísticamente significativa entre los grupos A y $B$ en las escalas CONSTANT y DASH en las evaluaciones previas a la cirugía como tampoco en el seguimiento a seis y 12 meses de los procedimientos, sólo en dos casos del grupo A $(11.7 \%)$ se requirieron ambos procedimientos. Conclusiones: La efectividad terapéutica de la punción y aspiración dirigida por ecocardiografía no resulta estadísticamente inferior al procedimiento artroscópico para resolver a la tendinitis calcificante del hombro.

Palabras clave: Tendinitis calcificante de hombro, punción y aspiración guiada por ultrasonido, artroscopia de hombro.

* Ortopedista. División de Cirugía Articular del Hospital de la Santa Creu I Sant Pau. Barcelona, España.

‡ Ortopedista. División de Cirugía Articular del Hospital Ángeles León, León, Guanajuato, México.

$\S$ Ortopedista. Universidad Autónoma de Barcelona. España.

Correspondencia:

Luis Gerardo Domínguez Gasca

Correo electrónico: luisdom88@hotmail.com

Aceptado: 01-05-2020.

www.medigraphic.com/actamedica

\section{Abstract}

Introduction: Shoulder calcic tendinopathy is one of the main causes of shoulder pain of non-traumatic origin in people between $50-60$ years of age, causing partial disability in the adult. Objective: To compare the therapeutic effectiveness of puncture and ultrasound-guided aspiration (group A) with arthroscopic repair of calcifying tendinitis (group B). Material and methods: 32 patients treated at the "Hospital de la Santa Creu i Sant Pau" in Barcelona, Spain, between february 2017 and february 2019; 17 patients formed group $A$ and 15 group $B$, assessing results with clinical and radiological improvement and scales of shoulder functionality (CONSTANT and DASH) performed prior to surgery, after six months and one year. Results: No statistically significant difference was found between groups A and B in the CONSTANT and DASH scales in the pre-surgery evaluations as well as in the six and 12 month follow-up of the procedures performed; only in two cases of group A $(11.7 \%)$ were both procedures required. Conclusions: Therapeutic effectiveness of puncture and aspiration guided by echocardiography is not statistically inferior to the arthroscopic procedure to resolve the shoulder calcifying tendonitis.

Keywords: Calcifying shoulder tendinitis, ultrasound-guided puncture and aspiration, shoulder arthroscopy.

\section{INTRODUCCIÓN}

La tendinopatía calcificante del manguito rotador (TC) se caracteriza por la presencia de depósitos de cristales de calcio en un tendón viable del manguito rotador del hombro, que usualmente mejora espontáneamente o es asintomática. ${ }^{1}$ La calcificación distrófica consiste en una acumulación de material cálcico que evoluciona a degeneración o ruptura tendinosa. ${ }^{2}$ Louwerens, $^{3}$ Dieh $^{4}$ y Oliva ${ }^{5}$ reportan que la patología ocurre más frecuentemente entre la quinta y la sexta década de la vida, con 
predominio en el género femenino con relación 1.5:1, con predilección en el tejido conjuntivo del supraespinoso en $50 \%$ de los casos en pacientes con sedentarismo (45\%). En 1907, Painter ${ }^{6}$ describió por primera ocasión el hallazgo radiológico de la TC en esa articulación esternoclavicular. En 1934, Codman ${ }^{7}$ concluye que la degeneración del tendón precede a la formación cálcica, así como también creía que los depósitos mayores a 15 mm de diámetro, eran los que generaban síntomas. En 1941, Bosworth, ${ }^{8}$ en estudio de 6,061 pacientes adultos realizando radiografías AP de ambos hombros, encontró una incidencia de $2.7 \%$ de depósitos de calcio adyacentes al troquíter en 51\% de los casos, pero sólo 35\% describieron algún tipo de sintomatología. Burkhead ${ }^{9}$ y Gohlke ${ }^{10}$ proponen que la aparición de esta patología se debe a un proceso degenerativo que involucra cambios necróticos de las fibras del tejido conectivo, que progresa a una acumulación distrófica de material cálcico. Mclaughlin ${ }^{11}$ menciona que ese depósito de cristales es precedido por hialinización focal de las fibras que se transforman en un patrón fibrilar y comienzan a desinsertarse del tendón 12 formando cuerpos de "arroz" que evolucionan a la calcificación.

\section{MATERIAL Y MÉTODOS}

Con protocolo aprobado por el Comité de Ética de la institución, se realizó un estudio de casos transversal, retrospectivo, analítico y operacional, con el objetivo de comparar la incisión y aspiración guiada por ecografía de la formación de depósitos cálcicos en el hombro, contra la realización de artroscopia en la resolución del problema. Para ello se invitó a participar a todo aquel paciente a quien se le pronunció el diagnóstico de tendinopatía cálcica; con aquellos que aceptaron participar en la investigación y firmaron carta de consentimiento informado, se conformaron dos conjuntos, el A, constituido por pacientes que acudieron al servicio de traumatología del Hospital de la Santa Creu i Sant Pau con diagnóstico de tendinitis calcificante, a los que se les realizó punción aspiración dirigida por eco; el conjunto B fue conformado por pacientes con diagnóstico de TC, a los que se les realizó cirugía artroscópica por el mismo problema. La muestra fue obtenida de manera no probabilística con selección intencional, de pacientes mayores de 20 años, independientemente de género y ocupación, evaluados y tratados por el servicio de traumatología entre febrero 2017 y febrero 2019, con un año de seguimiento mínimo.

Los criterios de inclusión fueron: pacientes con diagnóstico de TC a los que se les redactó historia clínica, en quienes no se hubiera efectuado punción aspiración conducida por ecografía ni operación artroscópica, con menos de un año de seguimiento posterior a la intervención.
Anotación de la fecha de punzadura aspiración guiada por ecografía o de la intervención artroscópica, incluyendo los datos del tamaño y localización del depósito, evaluando en consultas subsecuentes mejoría clínica y radiológica, basándose en radiografías y escalas de funcionalidad del hombro (CONSTANT y DASH) previo a la cirugía, a los seis meses y al año. Los criterios de exclusión fueron: pacientes que no cumplieran los criterios de diagnóstico o presentaran otra patología concomitante, así como aquellos con manifestaciones neurológicas o con problemas de enfermedades sistémicas que involucraran a la articulación escápulo-humeral. Dese el punto de vista estadístico, se utilizaron medidas de tendencia central para las variables continuas y de proporción para las nominales, utilizando $\chi^{2}$ para comparar resultados.

\section{RESULTADOS}

La muestra fue constituida por 32 pacientes acogidos en el Hospital de la Santa Creu i Sant Pau, atendidos en el periodo comprendido entre febrero de 2017 y febrero de 2019.

El grupo A consistió en 17 pacientes a los que se les realizó punción y aspiración guiada por ecografía, el B fue de 15 pacientes en los que se efectuó intervención artroscópica de hombro; se anota que del grupo A, en dos casos (11.7\%) se les realizaron ambos procedimientos. La distribución por género mostró $65.62 \%(n=21)$ al femenino y $34.38 \%(n$ $=11$ ) al masculino. La edad arrojó X y DE de $49.76 \pm 5.41$ años de edad, en relación al género el $X$ de 43 años de edad correspondió al femenino y de 51 años para el masculino. La lateralidad de la patología mostró preponderancia de

Tabla 1: Valoraciones en escalas de DASH
y CONSTANT de pacientes con tendinitis
calcificada de hombro, manejados por
punción aspiración vs artroscopia.



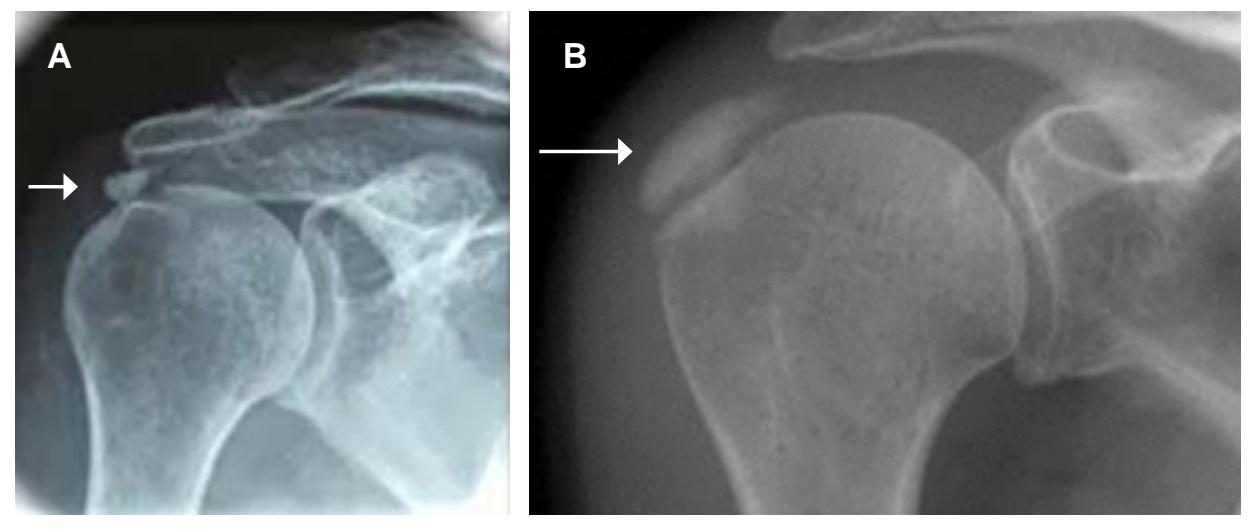

Figura 1:
A) Radiografía
anteroposterior de hombro derecho, mostrando calcificación bien delimitada del tendón del supraespinoso. B) Gran formación cálcica de límites difusos en el tejido conectivo del supraespinoso.

$92 \%$ en el hombro dominante y $8 \%$ para el contralateral, $(\mathrm{p}=0.001)$. El tendón afectado por la TC correspondió en $94.11 \%(n=30)$ al supraespinoso y $5.88 \%(n=2)$ al infraespinoso, $(p=0.0001)$. Con respecto al tamaño de la tendinitis calcificada se encontró: que $11.76 \%(n=4)$ eran pequeños; $35.29 \%(n=11)$ medianos y $52.94 \%(n=$ 17) grandes. En relación a la morfología de la acumulación de material cálcico se observó que el $64.7 \%(n=21)$ fue de tipo densa; $23.52 \%(\mathrm{n}=8)$ de tipo no calcificada y el $11.76 \%(n=4)$ de tipo nubosa. Los datos de valoración en escalas de DASH y CONSTANT se muestran en la Tabla 1, encontrando que no existió diferencia estadísticamente significativa entre los dos conjuntos, no obstante, llama la atención que los resultados a seis meses en el grupo A mostraron mejores cifras en ambas escalas, acercándose a niveles estadísticamente significativos.

\section{DISCUSIÓN}

Para clasificar a la TC se han propuestos diferentes métodos, en cuanto a la severidad de los síntomas en: aguda, sub aguda y crónica propuesta por Depalma ${ }^{13}$ en 1961; en 1988, Patte y Goutallier ${ }^{14}$ describieron una clasificación según la evolución en dos grupos: el tipo I con apariencia homogénea y resolución espontánea, y el tipo II con apariencia heterogénea caracterizado por cronicidad; desde el punto de vista de la acumulación de calcio, se mencionan 4 tipos: el tipo A con calcificación de bordes bien definidos y apariencia homogénea (Figura 1A); el tipo B de bordes difusos y apariencia homogénea (Figura 1B); el tipo $C$ de bordes bien definidos y apariencia heterogénea y; el tipo $\mathrm{D}$ de bordes difusos y apariencia heterogénea.

En relación a la patogenia de la enfermedad, a pesar de que es controvertida, Uhthoff y cols. ${ }^{15}$ describieron el ciclo de depósitos de calcio y la historia natural de la enfermedad; dividen la condición en dos fases, una formativa y otra resortiva, posterior a esto, varios autores mencionan que entre estas dos fases principales existen tres estadios que son: precalcificación o etapa silente, calcificación o pinzamiento y postcalcificación o agudo. La parte crónica de la primera fase (formativa) es ocasionada por hipoxia transitoria del tendón, asociada a microtraumas repetidos o a traumatismo directo simple, incrementándose los proteoglicanos que originan metaplasia de los tenocitos a condrocitos, induciendo la precipitación de calcio en la matriz extracelular hasta formar una masa.

Durante los estadios iniciales de la fase resortiva, histológicamente alrededor de los depósitos de calcio, existe vascularización con infiltración de macrófagos y células gigantes mononucleares y fibroblastos, ocasionando reacción inflamatoria agresiva, acumulación celular, edema excesivo y aumento de la presión intratendinosa, esto se manifiesta como incremento del dolor y pinzamientos, causado por al aumento del grosor de dicho tejido conjuntivo, que puede generar ruptura de los depósitos cálcicos al espacio subacromial ${ }^{2} y / 0$ a la bursa subacromial. ${ }^{14}$ Durante la etapa de postcalcificación los fibroblastos producen colágeno, principalmente tipo II, que va a rellenar el espacio dejado por la lesión, que al madurar se modifica a colágeno de tipo I entre 12 a 18 meses.

La presentación clínica de la TC dependerá, como muchas condiciones inflamatorias, de la fase en que se encuentre (aguda, sub aguda o crónica). En la fase aguda sintomática, el paciente acudirá con dolor severo de aparición súbita, generalmente atraumática, con moderada inflamación y disminución del rango de movimientos de ese fulcro. Al examen físico se puede observar al paciente con dolor evidente al realizar las pruebas exploratorias; en cuanto a diagnóstico diferencial debe desecharse un proceso infeccioso, que se descarta con la ausencia de fiebre en la mayoría de los casos, así como el síndrome de pinzamiento ocasionado por alteración del acromion. Durante las fases subagudas y crónicas, los síntomas dependen de la localización del depósito y del tamaño del mismo, es decir, a mayor dimensión de la lesión, más grandes serán los síntomas que irán desde un pinzamiento 
subacromial hasta un dolor difuso en dicha articulación esternoclavicular. ${ }^{14}$

Los estudios por imágenes confirman el diagnóstico, incluso en casos asintomáticos, y se usan también para realizar el seguimiento. Las radiografías AP verdadera de hombro, lateral de escápula y outlet de hombro, son las utilizadas para determinar la extensión, localización y diseminación de la lesión del supraespinoso. Las radiografías AP en rotación externa permiten valorar al subescapular, mientras que la imagen AP en rotación interna detecta acumulación de calcio en el redondo menor e infraespinoso. ${ }^{16}$ El ultrasonido diagnóstico muestra mayor sensibilidad en detectar calcificaciones en el manguito, con la desventaja de ser dependiente del operador. ${ }^{17} \mathrm{La}$ tomografía computarizada permite la localización más específica de los depósitos calcáreos, por otra parte el uso de la resonancia magnética es superior para determinar la existencia de otras lesiones y del estado del tendón ${ }^{18}$ (Figura 2).

La TC, así como sus tratamientos, han sido objeto de estudio desde hace ya más de 100 años, sin embargo no existe un consenso absoluto en el abordaje definitivo de esta patología. La TC se observa en ambos géneros, con moderada predilección por el femenino entre la tercera y la cuarta década de la vida, y es un problema ortopédico muy frecuente de tal articulación, concordando las observaciones de este trabajo con el reporte de Patte; ${ }^{14}$ a su vez, en cuanto al predominio de sintomatología en el hombro dominante este estudio concuerda con el reporte Uhthoff. ${ }^{15}$

Respecto al sitio donde se depositan los cristales de calcio, las observaciones de este reporte indican que 94.1 de los casos se presentaron en el tejido conectivo supraespinoso, a diferencia del estudio de Loew y colegas, 19,20 quienes localizaron la calcificación en $63 \%$ de los casos en el supraespinoso, $20 \%$ entre el tendón del supraespinoso y del subescapular, 3\% en este último, $7 \%$ en el infraespinoso y $7 \%$ en la bursa subacromial.

En relación a la dimensión de esa formación, el reporte de Bosworth y asociados ${ }^{8}$ indica que el mayor grupo corresponde al de pequeño tamaño con $60 \%$, seguido por el de extensión media con $25 \%$ y, por último, de magnitud grande con $15 \%$, revelando discrepancia con el presente estudio ya que el tamaño grande alcanzó $52.9 \%$ de los casos.

Respecto a la morfología de la formación cálcica, este estudio concuerda con el reporte de Gartner y colegas, en que el tipo denso de calcificación es el más frecuente.
Figura 2:

Imágenes de resonancia magnética de hombro derecho. A) imagen frontal oblicua, ponderada en T2 mostrando calcio dentro del tendón del supraespino (flecha), además de líquido con señal hiperintensa en la bolsa subacromial/ subdeltoidea (flecha curva).

B) imagen frontal oblicua mostrando señal hiperintensa de calcio dentro de ese ligamento del supraespinoso.
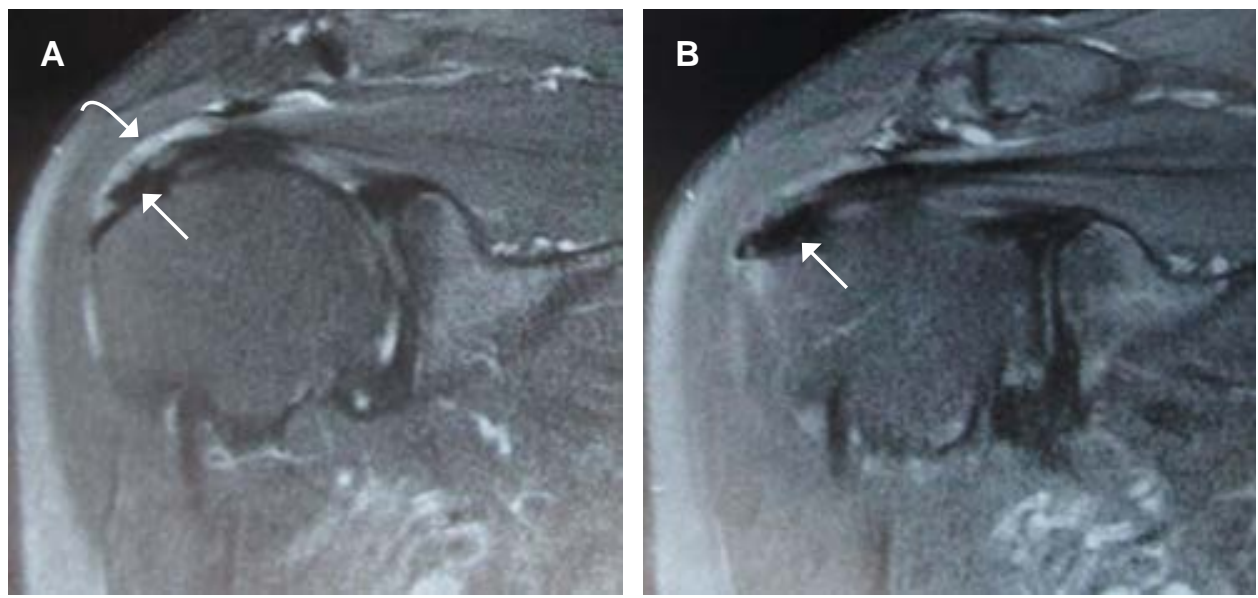
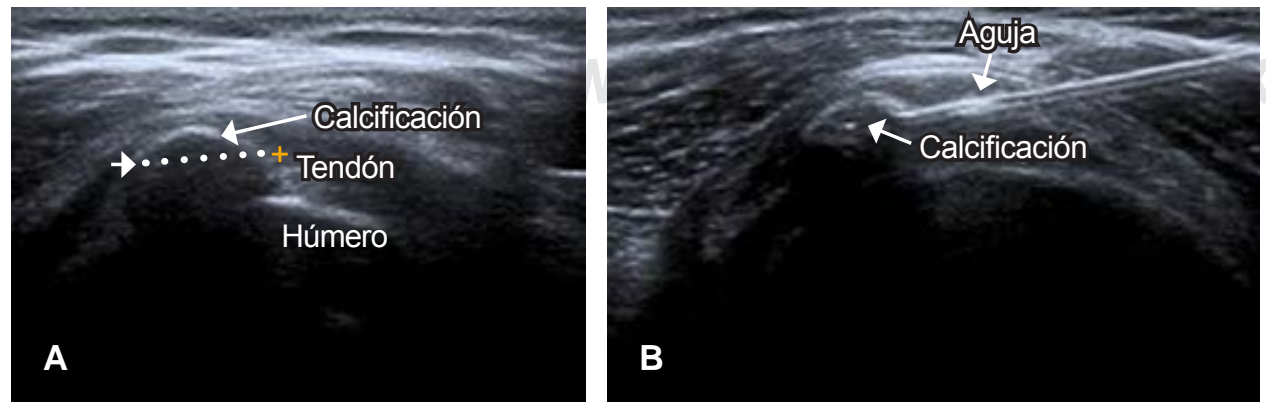

Figura 3:

Imágenes de ultrasonido diagnóstico mostrando en (A) imagen hipoecoica correspondiendo a calcificación del tendón del supraespinoso. B) Punción guiada por ultrasonido con aspiración de material cálcico en ese ligamento del supraespino. 
Figura 4:

Imágenes artroscópicas de hombro derecho con tendinopatía cálcica del manguito de los rotadores.
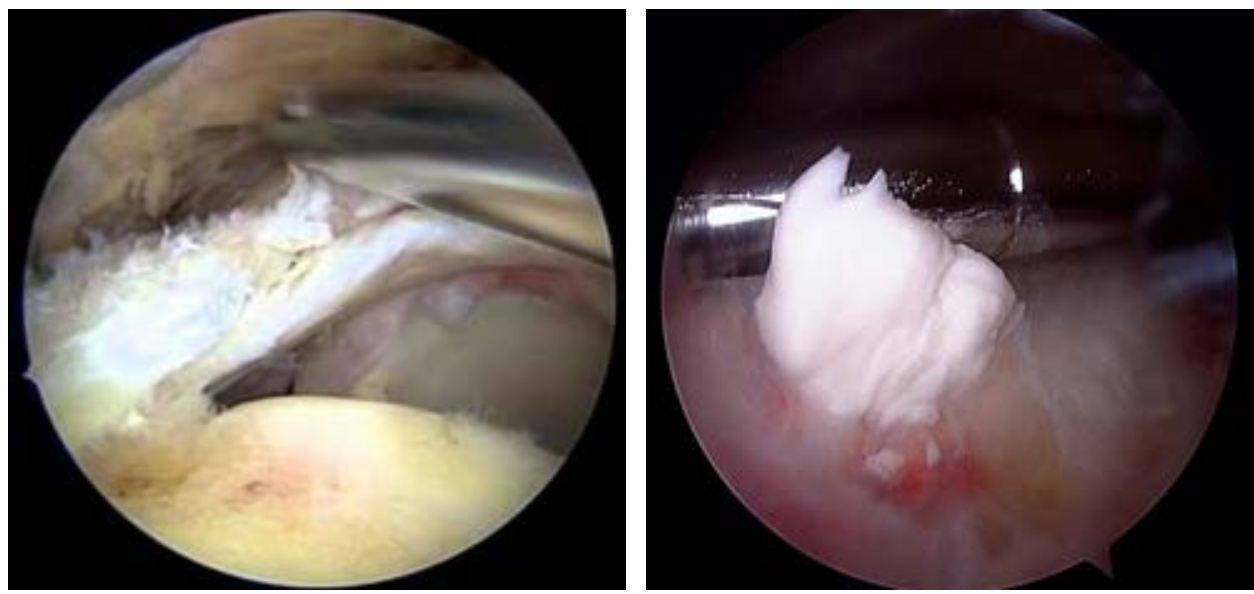

Las punciones de las formaciones cálcicas no son algo nuevo, en el año 1913, Flint describió esta técnica al igual que la reportó Codman ${ }^{7}$ en 1934. Desde los años 50 del siglo pasado, varios autores recomendaban las punciones a ciegas de los depósitos de calcio con una alta tasa de éxitos (85\%) defendiendo a la punzadura de la calcificación y no la exéresis de la misma. ${ }^{21,22}$ Depalma y Kruper $^{13}$ popularizaron el uso de punciones a ciegas sin identificación radiológica con buenos resultados (72\%). En el año 1996, Farin y cols. ${ }^{1}$ construyeron un estudio donde realizaron punciones y aspiraciones guiadas por ecografía demostrando mejoría de los síntomas en 75\% de los casos. En 2014, Gatt y asociados ${ }^{23}$ reportaron que el uso de punciones y aspiración genera mejoría de los síntomas en el 88\% de los pacientes. En 2013, De White ${ }^{24}$ comparó la punción aspiración dirigida por ultrasonido contra el uso de esteroides intralesionales (Figura 3), reportando que la primera tenía mejores resultados (83\%) que la segunda (63\%). Castillo y colaboradores ${ }^{12}$ además reportan que la incisión y aspiración conducida por ultrasonido, con un seguimiento de dos años, muestra la desaparición de la sintomatología en 98\% con evidencia radiológica de desaparición de la calcificación del $70 \%$.

En relación a desaparición de la sintomatología, en este trabajo 17 casos fueron sometidos a punciones y aspiración orientada por ecografía, de los cuales en dos casos $(11.7 \%)$ se requirió someterlos a artroscopia pues no presentaron mejoría. Al comparar estos resultados con el reporte de Castillo y colegas ${ }^{12}$ se revelaron mejores, ya que en sus casos reportados $18 \%$ requirió de intervención artroscópica (Figura 4).

Por último, tanto en la escala DASH como la CONSTANT, en esta investigación no se encontró diferencia estadísticamente significativa entre los grupos; no obstante, llama la atención que los resultados a seis meses en el grupo
A mostraron mejores cifras en ambas escalas, acercándose a niveles estadísticamente significativos.

\section{CONCLUSIONES}

De acuerdo con el estudio efectuado, el manejo de la tendinopatía cálcica de hombro debe inicialmente manejarse con punción y aspiración, pues resulta un método mínimamente invasivo, de bajo costo, con $88.3 \%$ de excelentes resultados, con respuesta de mejoría inmediata además de mantenerse en el tiempo, en contraste con el procedimiento artroscópico en donde la mejoría es progresiva, por lo que se puede concluir que la incisión aspiración de la tendinitis calcificada no es inferior al compararse con la cirugía artroscópica para la resolución de este problema frecuente.

\section{REFERENCIAS}

1. Farin $\mathrm{PU}$, Rasanen $\mathrm{H}$, Jaroma $\mathrm{H}$ et al. Rotator cuff calcifications: treatment with US-guided percutaneous needle aspiration and lavage. Skeletal Radiol. 1996; 25 (6): 551-554.

2. Uhthoff HK, Sarkar K. Classification and definition of tendinopathies. Clin Sports Med. 1991; 10 (4): 707-720.

3. Louwerens JK, Sierevelt IN, van Hove RP, van den Bekerom MP, van Noort A. Prevalence of calcific deposits within the rotator cuff tendons in adults with and without subacromial pain syndrome: clinical and radiologic analysis of 1,219 patients. J Shoulder Elbow Surg. 2015; 24 (10): 1588-1593.

4. Diehl P, Gerdesmeyer L, Gollwitzer H, Sauer W, Tischer T. Calcific tendinitis of the shoulder. Orthopade. 2011; 40 (8): 733-746.

5. Oliva F, Via AG, Maffulli N. Calcific tendinopathy of the rotator cuff tendons. Sports Med Arthrosc Rev. 2011; 19 (3): 237-243.

6. Painter C. Subdeltoid bursitis. Boston Med Surg J. 1907; 156: 345-349.

7. Codman EA. Calcified deposits in the supraspinatus. In: Cod- man EA. The shoulder: rupture of the supraspinatus tendon and other lesions in or about the subacromial bursa. Boston, MA: Thomas Todd. 1934, 78-215.

8. Bosworth BM. Examination of the shoulder for calcium deposits. J Bone Joint Surg. 1941; 23: 567-577. 
9. Burkhead WZ. A history of the rotator cuff before Codman. J Shoulder Elbow Surg. 2011; 20 (3): 358-362.

10. Gohlke F. Early European contributions to rotator cuff repair at the turn of the 20th century. J Shoulder Elbow Surg. 2011; 20 (3): 352-357.

11. Mclaughlin HL. The selection of calcium deposits for operation; the technique and results of operation. Surg Clin North Am. 1963; 43: 1501-1504.

12. Castillo-González FD, Ramos-Álvarez JJ, Rodríguez-Fabián G, González-Pérez J, Calderón-Montero J. Treatment of the calcific tendinopathy of the rotator cuff by ultrasound-guided percutaneous needle lavage. Two years prospective study. Muscles Ligaments Tendons J. 2014; 4 (2): 220-225.

13. Depalma AF, Kruper JS. Long-term study of shoulder joints afflicted with and treated for calcific tendinitis. Clin Orthop. 1961; 20: 61-72.

14. Patte D, Goutallier D. Periarthritis of the shoulder. Calcifications. Rev Chir Orthop Reparatrice Appar Mot. 1988; 74 (4): 277-278.

15. Uhthoff HK, Loehr JW. Calcific tendinopathy of the rotator cuff: pathogenesis, diagnosis, and management. J Am Acad Orthop Surg. 1997; 5 (4): 183-191.

16. Diehl P, Schauwecker J. Calcific tendinitis of the shoulder: operative and nonoperative treatment options. MMW Fortschr Med. 2014; 156 (17): 56-59.

17. Izadpanah K, Jaeger M, Maier D, Südkamp NP, Ogon P. Preoperative planning of calcium deposit removal in calcifying tendinitis of the rotator cuff - possible contribution of computed tomography, ultrasound and conventional X-Ray. BMC Musculoskelet Disord. 2014; 15: 385.

18. Filippucci E, Delle Sedie A, Riente L, Di Geso L, Carli L, Ceccarelli F et al. Ultrasound imaging for the rheumatologist. XLVII. Ultrasound of the shoulder in patients with gout and calcium pyrophosphate deposition disease. Clin Exp Rheumatol. 2013; 31 (5): 659-664.

19. Loew M, Sabo D, Wehrle M, Mau H. Relationship between calcifying tendinitis and subacromial impingement: a prospective radiography and magnetic resonance imaging study. J Shoulder Elbow Surg. 1996; 5 (4): 314-319.

20. Loew M, Jurgowski W, Mau HC, Thomsen M. Treatment of calcifying tendinitis of rotator cuff by extracorporeal shock waves: A preliminary report. J Shoulder Elbow Surg. 1995; 4 (2): 101-106.

21. Psaki CG, Carroll J. Acetic acid ionization; a study of determine the absorptive effects upon calcified tendinitis of the shoulder. Phys Ther Rev. 1955; 35 (2): 84-87.

22. Friedman MS. Calcified tendinitis of the shoulder. Am J Surg. 1957; 94 (1): 56-61.

23. Gatt DL, Charalambous CP. Ultrasound-guided barbotage for calcific tendonitis of the shoulder: a systematic review including 908 patients. Arthroscopy. 2014; 30: 1166-1172.

24. De Witte PB, Selten JW, Navas A, Nagels J, Visser CP, Nelissen RG, Reijnierse M. Calcific tendinitis of the rotator cuff: a randomized controlled trial of ultrasound-guided needling and lavage versus subacromial corticosteroids. Am J Sports Med. 2013; 41 (7): 1665-1673.

Financiamiento y conflicto de intereses: Los autores declaran no haber recibido patrocinio de ninguna índole en la elaboración de esta investigación, así mismo declaran no tener, ni existir conflicto de intereses. 